\title{
Childhood cancer survival rates in two South African units
}

\author{
D K Stones, ${ }^{1} \mathrm{MB} \mathrm{ChB}$, MMed, DCH; G P de Bruin, ${ }^{2} \mathrm{MB} \mathrm{ChB}$, MMed, FCPaed, DCH; T M Esterhuizen, ${ }^{3} \mathrm{MSc}$ (Epidemiol); \\ D C Stefan, ${ }^{2} \mathrm{MD}, \mathrm{MMed}, \mathrm{FCPaed}, \mathrm{CMO}, \mathrm{MSc}, \mathrm{PhD}$ \\ ${ }^{1}$ Department of Paediatrics and Child Health, Universitas Academic Hospital Complex, Faculty of Health Sciences, University of the Free State, \\ Bloemfontein, South Africa \\ ${ }^{2}$ Department of Paediatrics and Child Health, Faculty of Medicine and Health Sciences, Stellenbosch University, Tygerberg, Cape Town, \\ South Africa \\ ${ }^{3}$ Biostatistics Unit, Centre for Evidence-Based Health Care, Faculty of Medicine and Health Sciences, Stellenbosch University, Tygerberg, \\ Cape Town, South Africa
}

Corresponding author: D C Stefan (cs@sun.ac.za)

\begin{abstract}
Introduction. Childhood cancer is relatively rare, but there is a very good chance of cure. While overall survival rates of $>70 \%$ are reported from developed countries, survival is much less likely in developing countries and unknown in many countries in Africa.

Objective. To analyse survival rates of childhood cancers in two South African paediatric oncology units.

Methods. This retrospective review included all children ( 0 - 15 years) admitted with a malignancy at two paediatric oncology units (Universitas Hospital Academic Complex in Bloemfontein, Free State, and Tygerberg Hospital in Cape Town, Western Cape) between 1987 and 2011. The protocols used in the units were similar, and all the diagnoses were confirmed histologically.

Results. There were 3241 children, $53.5 \%$ of whom were males. Median follow-up was 17 months. The most common cancers were leukaemia (25.0\%), brain tumours (19.5\%), lymphoma (13.0\%) and nephroblastoma (10.0\%). The prevalences of neuroblastoma and retinoblastoma were similar at $5.8 \%$ and $5.7 \%$, respectively. Overall survival was calculated to be $52.1 \%$. Lymphoma and nephroblastoma had the highest survival rates at $63.9 \%$ and $62.6 \%$, respectively. Brain tumours had the lowest survival rate at $46.4 \%$. A comparison between ethnic groups showed white children to have the highest survival rate $(62.8 \%)$; the rate for children of mixed racial origin was $53.8 \%$ and that for black children $48.5 \%$.

Conclusions. Overall survival rates for children admitted to two paediatric cancer units in South Africa were lower than data published from developed countries, because many children presented with advanced disease. New strategies to improve cancer awareness are urgently required.
\end{abstract}

S Afr Med J 2014;104(7):501-504. DOI:10.7196/SAMJ.7882

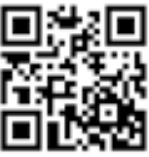

Childhood cancer is uncommon, representing $1-10 \%$ of all cancers reported globally. ${ }^{[1]}$ Globally, about 160000 new cases of childhood cancer are diagnosed annually, ${ }^{[2]} 13000$ in children and adolescents $(<20$ years) living in the USA. The annual average age-adjusted incidence rate in the USA is 158/million children. ${ }^{[3]}$ Most childhood cancers $(\sim 60 \%)$ occur in low- and middle-income countries where there is limited access to therapy, resulting in poor survival rates. ${ }^{[1,4]}$

Information about childhood cancer in Africa is lacking, as there are few formal cancer registries across the continent. The annual incidence of childhood cancer was estimated to be between 33.4 and 47.2/million between 2003 and 2007. ${ }^{[5]}$ The age-standardised annual incidence for all childhood malignancies has been estimated at 87.8 and 62.6/million in the Western Cape and Free State provinces of South Africa (SA), respectively (Stefan DC et al., childhood cancer incidence in South Africa, 1987 - 2007, unpublished data). Increased resources, improved care and ongoing research have resulted in a consistent improvement in survival of children with cancer in developed countries, with the 5-year survival rate of children and adolescents in the USA approaching $80 \%$ and a mortality rate of $2.8 / 100000 .^{[2]}$ Similar results are reported from Europe, with the 3 -year survival rate for all childhood cancers being $81 \%{ }^{[6]}$

Determinants of mortality and survival in children in the developing world (including Africa) differ markedly from those in the developed world. Survival rates of children with cancer in developing countries are far lower than those in the developed world. ${ }^{[4]}$ Deaths from paediatric cancer often go unreported, so the true mortality rate is unknown.

There are many obstacles to early diagnosis and effective treatment of childhood cancer in Africa. Limited resources, late presentation of disease, ${ }^{[7]}$ co-morbid infections (such as HIV) and malnutrition are among the barriers preventing improvement in survival

\section{Methods}

A retrospective demographic analysis of all children aged $0-15$ years diagnosed with a malignancy between 1 January 1987 and 31 December 2011 in two SA paediatric oncology units (POUs) (Universitas Hospital Academic Complex (UHAC) in Bloemfontein, Free State, and Tygerberg Hospital in Cape Town, Western Cape) was performed. Both units have dedicated POUs admitting children referred from the large surrounding areas they serve.

Tygerberg Hospital is a tertiary hospital located in Cape Town, serving the eastern metropolitan region of Cape Town and the north-eastern districts of the Western Cape. The hospital provides healthcare to over 3.6 million people (2.4 million children) and is the largest hospital in the Western Cape and the second-largest in SA. The POU admits on average 50 - 60 new patients per year, with an average hospital stay of 5 days per admission.

The Universitas Hospital Academic Complex (UHAC) in Bloemfontein provides cancer care to over 1.5 million children. It is the referral POU for the whole of the Free State and Northern Cape 
provinces, as well as some areas of both the North West and Eastern Cape provinces and Lesotho. This unit sees approximately 100 new oncology patients per year.

Data for this study were obtained from the children's tumour registries at the respective POUs and cross-checked with the South African Paediatric Tumor Registry. The registries of both units are similar, collect the same information and are part of the SA Paediatric Tumour Registry. Classification of childhood cancers in the registry is based on standards set by the International Classification of Childhood Cancers, 3rd edition. ${ }^{[8]}$

The protocols used in the two units were similar. The National Health Laboratory Service confirmed the histological diagnosis of malignancy in all cases where samples were available. In only a few cases (e.g. brainstem glioma) was diagnosis made on the clinical findings and relevant imaging, without histological studies.

The following variables were extracted and entered into a Microsoft Excel worksheet: date of birth, diagnosis, age at diagnosis, gender, ethnicity, HIV status, date last seen, and outcome at the end of the study.

SA has an ethnically diverse population, broadly classified as black, white and of mixed racial ancestry. The Western Cape population is $32.9 \%$ black, $48.8 \%$ mixed ancestry and $15.7 \%$ white (with $1 \%$ Asian/ Indian). In the Free State $87.6 \%$ are black, $3.1 \%$ of mixed ancestry, $8.7 \%$ white and $0.4 \%$ Asians/Indians. ${ }^{[9]}$

All children under 15 years of age were included. Disease was staged according to international guidelines. Survival was correlated with the stage of the disease and the ethnic group to which each patient belonged.

The five most common cancers were leukaemia, brain tumours, lymphoma, nephroblastoma and retinoblastoma. Nephroblastoma and retinoblastoma were further analysed with regard to survival rates, stage and patient ethnic groups. The staging of nephroblastoma was based on abdominal ultrasound and computed tomography (CT) of the abdomen and, if indicated, of the chest. Magnetic resonance imaging (MRI) was done in selected cases to facilitate surgery. The staging of retinoblastoma included ophthalmological examination, cerebrospinal fluid (CSF) analysis, bone marrow examination and a brain CT scan or MRI.

All children were followed up from the date of diagnosis until 31 December 2012, or until they died or were lost to follow-up. Outcomes were defined as overall survival at 1, 3 and 5 years. The dates on which patients died or were last seen were documented. Patients who were lost to follow-up, either while on treatment or after treatment, were included in the analysis as censored observations.

Patients from the two POUs were analysed both together and separately, to compare survival rates for the most common malignancies encountered. Kaplan-Meier curves were constructed for each comparison, and where possible median survival time and 95\% confidence intervals (CIs) were reported. Where cumulative survival probability did not drop below 50\%, mean survival times were reported. Log-rank tests were used to compare groups in terms of survival times. All calculations were performed using SPSS software (IBM SPSS Statistics, version 21). A $p$-value of $<0.05$ was considered statistically significant.

Ethics approval was obtained from the human ethics research committees of the two participating centres. Confidentiality was maintained by using de-identified data throughout.

\section{Results}

The initial database included 3292 children diagnosed with malignancy from both units. Excluded from the analysis were ten children who were missing at the end-date of their follow-up period, so survival periods could not be calculated; 36 who died before receiving treatment or had no outcome information; one whose ethnic background was not recorded, and four for whom data were incomplete. A total of 3241 children were therefore included in the study. There were 1735 males (53.5\%) and 1506 females (46.5\%) in the combined group ( $p=0.67)$. Of the children, $1449(44.7 \%)$ were aged 0 - 4 years, $962(29.7 \%) 5$ - 9 years, 808 (24.9\%) 10 - 14 years and $22(<1 \%)>14$ years.

The median duration of follow-up was 17 months (range 1 day 26.4 years). Between 1 January 1987 and 31 December 2012 in both POUs, 1551 children died from cancer or cancer-related infection or toxicity, giving an overall survival rate of $52.1 \%$ (median survival time 45.7 months; 95\% CI 29.9 - 61.5).

There was a $67.8 \%$ 1-year survival probability overall, with a median survival time of 9.3 months (95\% CI $9.16-9.46)$. At 3 years this had changed to $56.4 \%$ (mean 22.9 months; 95\% CI $22.4-23.5$ ), and at 5 years to $53.8 \%$ (mean 34.8 months; 95\% CI 33.9 - 35.8). Survival in all age groups was similar $(p=0.43)$.

The five most common malignancies were the same in the two units and echoed the pattern of the SA Paediatric Tumour Registry. In both units lymphoma and nephroblastoma had the highest survival rates at $63.9 \%$ and $62.6 \%$, respectively. Brain tumours had the lowest survival rate at $46.4 \%$ (Table 1 ).

The overall survival rates in the two units were similar $(55.8 \%$ for Tygerberg Hospital, 50.6\% for UHAC), with a combined overall rate of $52.1 \%$.

Over half the children were black ( $n=1886,58.2 \%)$; followed by children of mixed ancestry $(n=829,25.6 \%)$ and white children ( $n=522,16.1 \%)$ (ethnic group was known for 3237 children). Cancer deaths were significantly more common among children in the former two groups than among white children, with black children having the shortest mean survival time $(p<0.001)$. Survival was best

Table 1. Prevalences and survival rates for the most common malignancies in children in two paediatric oncology units in SA

\begin{tabular}{|c|c|c|c|c|}
\hline \multirow[b]{2}{*}{ Malignancy } & \multicolumn{2}{|c|}{ Prevalence, $n(\%)$} & \multirow[b]{2}{*}{$\begin{array}{l}\text { Overall survival } \\
n(\%)\end{array}$} & \multirow[b]{2}{*}{$\begin{array}{l}\text { Median surviva } \\
\text { time (months) }\end{array}$} \\
\hline & $\begin{array}{l}\text { UHAC } \\
(N=2284)\end{array}$ & $\begin{array}{l}\text { Tygerberg Hospital } \\
(N=957)\end{array}$ & & \\
\hline Leukaemia $(N=762)$ & $490(21.5)$ & $272(28.4)$ & $372(48.8)$ & 46.5 \\
\hline Brain tumours $(N=627)$ & $437(19.1)$ & $190(19.9)$ & $291(46.4)$ & 18.2 \\
\hline Lymphoma $(N=416)$ & $282(12.3)$ & $134(14.0)$ & $266(63.9)$ & Mean $185.3^{*}$ \\
\hline Nephroblastoma $(N=329)$ & $253(11.1)$ & $76(7.9)$ & $206(62.6)$ & 308.0 \\
\hline Retinoblastoma $(N=182)$ & $155(6.8)$ & $27(2.8)$ & $86(47.3)$ & 20.5 \\
\hline
\end{tabular}


among white children $(62.8 \%)$, followed by children of mixed ancestry $(53.8 \%)$ and black children (48.5\%).

Similar differences in survival times were found for all the five most common malignancies, with black children doing consistently least well (Table 2).

The overall survival rate for nephroblastoma (all stages) was 62.6\%. Survival for stage 1 nephroblastoma (81.1\%) was the best of all the cancer types, with a mean of 220 months. Children with disease at the other stages did progressively worse, and survival for stage IV disease was only $45.6 \%$. Differences in survival times between the stages were highly significant $(p<0.001)$. Black children presented predominantly with stage 3 and 4 disease. Analysis of survival between ethnic groups in different stages of disease demonstrated that black children had a significantly lower survival for all except stage 1 disease (log-rank test adjusted for stage, $7.4 ; p=0.02$ ) (Table 3 ).

Patients with retinoblastoma were divided into two groups, depending on whether they had early or late-stage disease (early defined as limited local, intraorbital involvement, and late as involvement of bone marrow and/ or CSF, and/or with brain metastases). Early and late stages of retinoblastoma, occurring in equal numbers in both units (90 v. 92), had survival rates of $84.4 \%$ and $10.9 \%$, respectively (overall survival $46.3 \%$ ). Black children presented predominantly with latestage disease $(56.9 \%$ ), as opposed to $29 \%$ of children of mixed ancestry and $14.3 \%$ of white children. Although sample sizes were small, black children demonstrated a nonsignificant trend towards a lower survival with stage 1 disease $(82.3 \%)$ v. $100 \%$ of white and $86.4 \%$ of mixed-ancestry patients.

\section{Discussion}

This is the first study of survival rates for children with cancer in SA. The results demonstrate that overall survival in childhood cancer remains low (52.1\%) when compared with international data. In the past 40 years, the overall survival rate for childhood cancer in the UK has increased from $10 \%$ to nearly $90 \% \cdot{ }^{[6,10]}$ The five most common malignancies in our cohort were similar to those commonly found in children in developed countries, ${ }^{[1]}$ but survival rates were lower.

Black children consistently had worse survival rates than other ethnic groups for all the five most common malignancies and in almost all stages of nephroblastoma and retinoblastoma (Fig. 1). No differences in survival were found between the genders or between different age groups. Possible

Table 2. Comparison of survival rates for the five most common childhood malignancies between three ethnic groups

\begin{tabular}{llll}
\hline & \multicolumn{3}{c}{ Children who survived, $\boldsymbol{n} / \mathbf{N}(\%)$} \\
\cline { 2 - 4 } Malignancy & Black & Mixed race & White \\
\hline Leukaemia $(N=762)$ & $151 / 371(40.7)$ & $115 / 212(54.2)$ & $106 / 179(59.2)$ \\
Brain tumours $(N=627)$ & $146 / 340(42.9)$ & $88 / 187(47.1)$ & $57 / 100(57.0)$ \\
Lymphoma $(N=415)$ & $140 / 240(58.3)$ & $82 / 112(73.2)$ & $43 / 63(68.3)$ \\
Nephroblastoma $(N=329)$ & $131 / 225(58.2)$ & $49 / 70(70.0)$ & $26 / 34(76.5)$ \\
Retinoblastoma $(N=182)$ & $61 / 144(42.2)$ & $19 / 31(61.3)$ & $6 / 7(85.7)$
\end{tabular}

Table 3. Nephroblastoma survival according to ethnic group and stage

\begin{tabular}{llll}
\hline & \multicolumn{3}{c}{ Children who survived, $\boldsymbol{n} / \boldsymbol{N}(\%)$} \\
\cline { 2 - 4 } Nephroblastoma $(\boldsymbol{N = 3 2 1})^{*}$ & Black & Mixed race & White \\
\hline Stage $1(N=95)$ & $46 / 56(82.1)$ & $19 / 25(76.0)$ & $12 / 14(85.7)$ \\
Stage $2(N=42)$ & $19 / 29(65.5)$ & $6 / 9(66.7)$ & $4 / 4(100.0)$ \\
Stage $3(N=92)$ & $36 / 67(53.7)$ & $13 / 17(76.5)$ & $5 / 8(62.5)$ \\
Stage $4(N=79)$ & $26 / 62(41.3)$ & $7 / 12(58.3)$ & $3 / 5(60.0)$ \\
*Stage $5(n=13)$ excluded. Data on stage missing $n=8$. & &
\end{tabular}

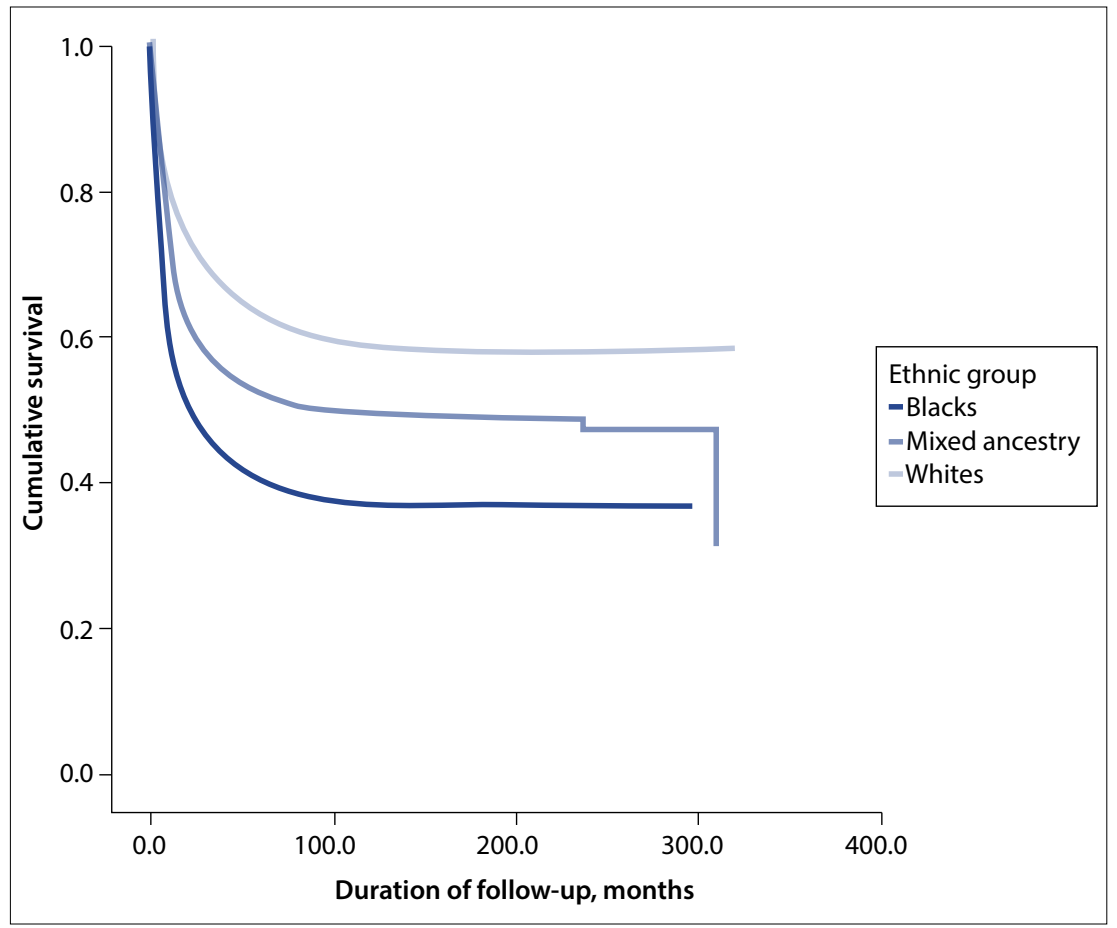

Fig. 1. Comparative cancer survival in children according to ethnic group.

reasons for these differences in outcome for the different groups include late presentation, poor nutritional status, genetic factors and associated comorbidities (e.g. HIV infection and tuberculosis). ${ }^{[12]}$

Children from low-income countries are often malnourished when a malignancy is diagnosed. Malnutrition in children with cancer renders them vulnerable to chemotherapy-related toxicity and infections, and ultimately increases the risk of death. Many studies document inadequate nutrition in black and coloured (mixed ancestry) children in SA, which is reflected in high rates of stunting and underweight. ${ }^{[13]}$ Oelofse et al. ${ }^{[13]}$ also found a substantially higher prevalence of micronutrient deficiencies among disadvantaged black and coloured infants in urban communities in the Western Cape than in other groups. All the above factors may result in a worse outcome for black children. 
During the study period, 23 HIV-infected children with a malignancy presented to Tygerberg Hospital and 93 to UHAC, giving a prevalence of HIV-infected children with a malignancy of $2.4 \%$ in the former and $4.05 \%$ in the latter. Many social and demographic factors differ in the various ethnic groups, profoundly affecting survival rates. These include employment-related issues, transport-associated factors, caregivers, traditional attitudes to cancer and other ethnic beliefs.

The fact that most black children with nephroblastoma and retinoblastoma presented at an advanced stage of disease is attributable to the few points at which to access care in low socioeconomic areas, late referral from the primary health sector, and little knowledge about paediatric malignancy on the part of healthcare workers, parents and the community as a whole. A previous study in the Tygerberg Hospital referral area showed that a considerable delay in diagnosis could be attributed to physicians making a wrong initial diagnosis, delay in performing clinical tests, and delay in obtaining histopathological results. ${ }^{[14]}$ Children in lower socioeconomic areas have less access to care than those in higher-income groups. Awareness campaigns in SA can improve knowledge about childhood malignancies and facilitate early diagnosis, particularly among these underprivileged children. However, stage of disease at presentation on its own cannot account for the differences in survival between the ethnic groups, black children showing significantly poorer survival at all stages of nephroblastoma (except stage 1) and retinoblastoma. It has been shown that children with cancer in low-income countries commonly fail to complete treatment. ${ }^{[15]}$ Abandonment of therapy was not a significant factor in our study, as most of the patients from distant areas were provided with lodging by the CHOC (Childhood Cancer Foundation South Africa). Notably, in the cohort attending Tygerberg Hospital, with a geographically smaller referral area, no cases were lost to follow-up.

Differences in outcome between the various ethnic groups have also been demonstrated in SA children with Hodgkin's lymphoma. ${ }^{[16]}$ Further research into ethnic differences is needed to investigate such survival discrepancies.

\section{Study limitations}

There are number of limitations to this study. It was retrospective, so we were unable to ascertain the full impact of factors such as nutrition on survival. Data on nutritional status at presentation were not collected, and nutritional influence on survival is an aspect demanding further attention and research.

These results, from only two POUs, cannot be extrapolated to the broader SA population, as some units have only had a comprehensive tumour registry since 1987. It is estimated that two-thirds of childhood malignancies in SA are not reported to the registry, either because they are not diagnosed or because the patient is not referred to an appropriate treatment centre. ${ }^{[17]}$

Sufficient staging information to perform a comparison was only available for two of the five most common malignancies. Further studies comparing staging information with survival for other solid tumours would be of interest.

\section{Conclusion}

Survival rates for childhood cancer in SA remain low when compared with international data. Children of black ethnic origin had significantly lower overall survival for all malignancies. Greater awareness of the danger signs of paediatric malignancy will foster earlier diagnosis of disease and lead to improved outcomes for all ethnic groups.

Author contributions. DKS collected the data in one POU, did the interpretation, and contributed to the discussion and editing of the paper. GDB wrote the protocol, collected the data in the other POU, and wrote the first draft of the paper. TME performed the statistical analysis and contributed to editing and discussions. DCS designed the study, and contributed to the writing and editing. All authors read and approved the manuscript.

\section{References}

1. Ferlay J, Shin HR, Bray F, et al. Estimates of worldwide burden of cancer in 2008: GLOBOCAN 2008 Int J Cancer 2010;127(12):2893-2917. [http://dx.doi.org/10.1002/ijc.25516]

2. Ribeiro R. Improving survival of children with cancer worldwide: The St. Jude International Outreach Program approach. Stud Health Technol Inform 2012;172:9-13. [http://dx.doi.org/10.3233/978-1 61499-088-8-9]

3. Linabery AM, Johnson KJ, Ross JA. Childhood cancer incidence trends in association with US folic acid

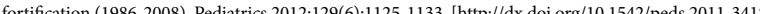

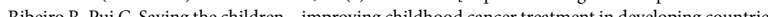
Nibeiro R, Pic C.

5. Stefan DC. Epidemiology of childhood cancer and the SACCSG tumour registry. Continuing Medical Education 2010;28(7):317-319.

6. Gatta G, Botta L, Rossi S, et al. Childhood cancer survival in Europe 1999-2007: Results of EUROCARE-5 - a population-based study. Lancet Oncol 2014;15(1):35-47. [http://dx.doi.org/10.1016 S1470-2045(13)70548-5]

7. Magnani C, Pastore G, Coebergh JW, et al. Trends in survival after childhood cancer in Europe, 1978 1997: Report from the Automated Childhood Cancer Information System project (ACCIS). Eur Cancer 2006;42(13):1981-2005. [http://dx.doi.org/10.1016/j.ejca.2006.05.006]

8. Steliarova Foucher E, Stiller C, Lacour B, Kaatsch P. International Classification of Childhood Cancer third edition. Cancer 2005;103(7):1457-1467. [http://dx.doi.org/10.1002/cncr.20910]

Statistics South Africa. http://www.statssa.gov.za/Publications/P03014/P030142011.pdf (accessed 5 December 2013)

10. Pritchard-Jones $\mathrm{K}$, Pieters R, Reaman GH, et al. Sustaining innovation and improvement in the treatment of childhood cancer: Lessons from high-income countries. Lancet Oncol 2013:14(3):e95-e103. [http://dx doi.org/10.1016/S1470-2045(13)70010-X]

11. CureSearch for Children's Cancer. http://www.curesearch.org/Childhood-Cancer-Statistics/ (accessed 2 March 2014)

12. Sinfield RL, Molyneux EM, Banda K, et al. Spectrum and presentation of pediatric malignancies in the HIV era: Experience from Blantyre, Malawi, 1998-2003. Pediatr Blood Cancer 2007;48(5):515-520. [http://dx.doi.org/10.1016/S1470-2045(13)70010-X]

13. Oelofse A, Van Raaij JM, Benadé AJ, et al. Disadvantaged black and coloured infants in two urban communities in the Western Cape, South Africa differ in micronutrient status. Public Health Nut 2002;5(2):289-294. [http://dx.doi.org/10.1079/PHN2002263]

4. Stefan D, Siemonsma F. Delay and causes of delay in the diagnosis of childhood cancer in Africa. Pediatr Blood Cancer 2011;56(1):80-85. [http://dx.doi.org/10.1002/pbc.22714]

15. Arora RS, Eden T, Pizer B. The problem of treatment abandonment in children from developin countries with cancer. Pediatr Blood Cancer 2007;49(7):941-946. [http://dx.doi.org/10.1002/pbc.21127]

6. Stefan D, Stones D, Dippenaar A, et al. Ethnicity and characteristics of Hodgkin lymphoma in children. Pediatr Blood Cancer 2009;52(2):182-185. [http://dx.doi.org/10.1002/pbc.21804]

17. Stefan DC, Stones D. The South African Paediatric Tumour Registry - 25 years of activity. S Afr Med J 2012;102(7):605-606

Accepted 11 April 2014. 